\title{
ON GRADED WEAK MULTIPLICATION MODULES
}

\author{
F. FARZALIPOUR AND P. GHIASVAND
}

\begin{abstract}
Let $G$ be a group with identity $e$, and let $R$ be a $G$-graded commutative ring, and let $M$ be a graded $R$-module. In this paper we characterize graded weak multiplication modules.
\end{abstract}

\section{Introduction}

Let $G$ be a group. A commutative ring $(R, G)$ is called a $G$-graded commutative ring if there exists a family $\left\{R_{g}: g \in G\right\}$ of additive subgroups of $R$ such that $R=\bigoplus_{g \in G} R_{g}$ such that $1 \in R_{e}$ and $R_{g} R_{h} \subseteq R_{g h}$ for each $g$ and $h$ in $G$. For simplicity, we will denote the graded ring $(R, G)$ by $R$. If $R$ is $G$-graded, then an $R$-module $M$ is said to be $G$-graded if it has a direct sum decomposition $M=\bigoplus_{g \in G} M_{g}$ such that for all $g, h \in G ; R_{g} M_{h} \subseteq M_{g h}$. An element of some $R_{g}$ or $M_{g}$ is said to be homogeneous element of degree $g$. A submodule $N \subseteq M$, where $M$ is G-graded, is called $G$-graded if $N=\bigoplus_{g \in G}\left(N \cap M_{g}\right)$ or if, equivalently, $N$ is generated by homogeneous elements. Moreover, $M / N$ becomes a $G$-graded module with $g$-component $(M / N)_{g}=\left(M_{g}+N\right) / N$ for $g \in G$. We write $h(R)=\cup_{g \in G} R_{g}$ and $h(M)=\cup_{g \in G} M_{g}$. A graded commutative ring $R$ is called graded integral domain, if $a b=0$ for $a, b \in h(R)$, then $a=0$ or $b=0$. A graded commutative ring $R$ is called graded field, if every homogeneous element of $R$ is unit. A graded ideal $I$ of $R$ is said to be graded prime ideal if $I \neq R$; and whenever $a b \in I$, we have $a \in I$ or $b \in I$, where $a, b \in h(R)$ (see, [6]). A graded ideal $I$ of $R$ is said to be graded maximal if $I \neq R$ and there is no graded ideal $J$ of $R$ such that $I \mp J \subsetneq R$. If $R$ is a graded ring and $M$ a graded $R$-module, the subset $T(M)$ of $M$ is defined by $T(M)=\{m \in M: r m=0$ for some $0 \neq r \in h(R)\}$. If $R$ is a graded integral domain, then $T(M)$ is a graded submodule of $M$ (see [1]). If $T(M)=0, M$ is called graded torsion free, if $T(M)=M, M$ is called graded torsion. A graded module $M$ over a $G$-graded $\operatorname{ring} R$ is called to be graded multiplication if for each graded submodule $N$ of $M ; N=I M$ for some graded ideal $I$ of $R$. One can easily show that if $N$ is graded submodule of a graded multiplication module $M$, then $N=(N: M) M$ [see, 4]. Let $R$ be a $G$-graded ring and $S \subseteq h(R)$ be a multiplicatively closed subset of $R$. Then

Corresponding author: Peyman Ghiasvand.

2010 Mathematics Subject Classification. 13C05, 13C13, 13 A15.

Key words and phrases. Minimal, Graded, graded prime, graded weak multiplication. 
the ring of fraction $S^{-1} R$ is a graded ring which is called the graded ring of fractions. Indeed, $S^{-1} R=\bigoplus_{g \in G}\left(S^{-1} R\right)_{g}$ where $\left(S^{-1} R\right)_{g}=\left\{r / s: r \in h(R), s \in S\right.$ and $g=(\text { deg } s)^{-1}($ deg $\left.r)\right\}$.

Let $M$ be a graded module over a $G$-graded ring $R$ and $S \subseteq h(R)$ be a multiplicatively closed subset of $R(0 \notin S, 1 \in S$ and for any $a, b \in S, a b \in S)$. The module of fraction $S^{-1} M$ over a graded ring $S^{-1} R$ is a graded module which is called the module of fractions, if $S^{-1} M=$ $\bigoplus_{g \in G}\left(S^{-1} M\right)_{g}$ where $\left(S^{-1} M\right)_{g}=\left\{m / s: m \in M, s \in S\right.$ and $g=(\text { deg } s)^{-1}($ deg $\left.m)\right\}$. We write $h\left(S^{-1} R\right)=\bigcup_{g \in G}\left(S^{-1} R\right)_{g}$ and $h\left(S^{-1} M\right)=\bigcup_{g \in G}\left(S^{-1} M\right)_{g}$. Let $M=\bigoplus_{g \in G} M_{g}$ and $N=\bigoplus_{g \in G} N_{g}$ are two graded modules, then a mapping $\eta: M \longrightarrow N$ is graded homomorphism if $\eta\left(M_{g}\right) \subseteq N_{g}$ for all $g \in G$. Consider the graded homomorphism $\eta: M \longrightarrow S^{-1} M$ defined by $\eta(m)=m / 1$. For any graded submodule $N$ of $M$, the submodule of $S^{-1} M$ generated by $\eta(N)$ is denoted by $S^{-1} N$. Similar to non graded case, one can prove that $S^{-1} N=\left\{\beta \in S^{-1} M: \beta=m / s\right.$ for $m \in N$ and $s \in S\}$ and that $S^{-1} N \neq S^{-1} M$ if and only if $S \cap(N: M)=\phi$. Let $P$ be any graded prime ideal of a graded ring $R$ and consider the multiplicatively closed subset $S=h(R)-P$. We denote the graded ring of fraction $S^{-1} R$ of $R$ by $R_{P}^{g}$ and we call it the graded localization of $R$. This ring is graded local with the unique graded maximal $S^{-1} P$ which will be denoted by $P R_{P}^{g}$. Moreover, $R_{P}^{g}$-module $S^{-1} M$ is denoted by $M_{P}^{g}$. For graded submodules $N$ and $K$ of $M$, if $N_{P}^{g}=K_{P}^{g}$ for every graded prime (graded maximal) ideal $P$ of $R$, then $N=K$.

Moreover, similar to non graded case, we have the following properties for graded submodules $N$ and $K$ of $M$ :

(1) $S^{-1}(N \cap K)=S^{-1} N \cap S^{-1} K$.

(2) $S^{-1}(N: K)=\left(S^{-1} N: S^{-1} K\right)$ if $K$ is finitely generated.

If $K$ is a graded submodule of $S^{-1} R$-module $S^{-1} M$, then $K \cap M$ will denote the graded submodule $\eta^{-1}(K)$ of $M$. Moreover, similar to the non graded case one can prove that $S^{-1}(K \cap$ $M)=K$. In this paper, we study properties of graded prime submodules and define graded weak multiplication module. Also, we characterize graded weak multiplication modules.

\section{Graded prime submodules}

A proper graded submodule $N$ of a graded $R$-module $M$ is defined to be graded prime if $r m \in N$ where $r \in h(R)$ and $m \in h(M)$, then $m \in N$ or $r \in(N: M)$ (see [3]).

The set of all graded prime submodules of a graded $R$-module $M$ is denoted by $G \operatorname{Spec}(M)$.

The following Lemma is known, but we write it here for the sake of references.

Lemma 2.1. Let $M$ be a graded module over a graded ring $R$. Then the following hold:

(i) If I and $J$ are graded ideals of $R$, then $I+J$ and $I \cap J$ are graded ideals. 
(ii) If $N$ is a graded submodule, $r \in h(R)$ and $x \in h(M)$, then $R x, I N$ and $r N$ are graded submodules of $M$.

(iii) If $N$ and $K$ are graded submodules of $M$, then $N+K$ and $N \cap K$ are also graded submodules of $M$ and $\left(N:_{R} M\right)$ is a graded ideal of $R$.

(iv) Let $N_{\lambda}$ be a collection of graded submodules of $M$. Then $\sum_{\lambda} N_{\lambda}$ and $\cap_{\lambda} N_{\lambda}$ are graded submodues of $M$.

Theorem 2.2. Let $R$ be a graded ring and $M \neq 0$ a graded $R$-module, then $R$ is a graded field if and only if every proper graded submodule of $M$ is a graded prime submodule of $M$ and $T(M) \neq M$.

Proof. $(\Rightarrow)$ Let $N$ be a proper graded submodule of $M$. Suppose that $r_{g} m_{h} \in N$ where $r_{g} \in h(R)$ and $m_{h} \in h(M)$. If $r_{g}=0$, then $r_{g}=0 \in(N: M)$, so let $r_{g} \neq 0$. Hence $m_{h}=r_{g}^{-1}\left(r_{g} m_{h}\right) \in N$ since $R$ is graded field. So $N$ is a graded prime submodule.

Now we show that $T(M) \neq M$. Let $T(M)=M$, and $0 \neq m \in M$. Then $r_{g} m=0$ for some $0 \neq r_{g} \in h(R)$. So $m=0$ since $R$ is graded field, a contradiction.

$(\Leftarrow)$ Let $m_{g} \in M-T(M)$, so $A n n\left(m_{g}\right)=0$. It is easy to see that every proper graded submodule of $R m_{g}$ is a graded prime submodule, and $R m_{g} \cong R / A n n\left(m_{g}\right)=R$ as graded $R$-modules. Therefore, every proper graded ideal of $R$ is a graded prime ideal, hence $R$ is a graded field (see, [2]).

Lemma 2.3. Let $M$ be a graded $R$-module, $S \subseteq h(R)$ a multiplicatively closed set and $N$ a graded prime submodule of $M$ such that $S \cap(N: M)=\varnothing$. Then $S^{-1}(N: M)=\left(S^{-1} N: S^{-1} M\right)$.

Proof. Let $r / s \in\left(S^{-1} N: S^{-1} M\right)$. Let $m \in M$, so $m=\sum_{g \in G} m_{g}$. We show that for any $g \in G$, $r m_{g} \in N$. $r / s . m_{g} / 1 \in S^{-1} N$. Hence $r m_{g} / s=n / t$ for some $n \in N$ and $t \in S$. So $s^{\prime} t r m_{g}=$ $s s^{\prime} n \in N$ for some $s^{\prime} \in S$ and since $s^{\prime} t \notin(N: M)$, so $N$ graded prime submodule gives $r m_{g} \in N$. Therefore, $r m \in N$, so $\left(S^{-1} N: S^{-1} M\right) \subseteq S^{-1}(N: M)$. It is clear that $S^{-1}(N: M) \subseteq\left(S^{-1} N: S^{-1} M\right)$. Thus $S^{-1}(N: M)=\left(S^{-1} N: S^{-1} M\right)$.

Theorem 2.4. Let $P$ be a graded prime ideal of $R, S \subseteq h(R)$ a multiplicatively closed set such that $S=h(R)-P$ and $M$ a graded $R$-module. Then there exists a one-to-one correspondence between the graded $P$-prime submodules of $M$ and the $P R_{p}^{g}$-prime $R_{P}^{g}$-submodules of $M_{p}^{g}$.

Proof. Let $N$ be a graded $P$-prime submodule of $M$. suppose $r / s . m / t \in N_{P}^{g}$ and $m / t \notin N_{P}^{g}$ where $r / s \in h\left(R_{P}^{g}\right)$ and $m / t \in h\left(M_{P}^{g}\right)$. Hence $r m \in N$ and $m \notin N$ where $r \in h(R)$ and $m \in h(M)$, so $r \in(N: M)$ since $N$ is graded prime. Hence $r / s \in\left(N:_{R} M\right)_{P}^{g}=\left(N_{P}^{g}:_{R_{P}^{g}} M_{P}^{g}\right)=P R_{P}^{g}$ by Lemma 2.3. Hence $N_{P}^{g}$ is a graded $P R_{P}^{g}$-prime $R_{P}^{g}$-submodule of $M_{P}^{g}$. Let $N_{P}^{g}$ be a graded prime submodule of $M_{P}^{g}$. Let $r m \in N$ where $r \in h(R)$ and $h(M)$. So $r / 1 . m / 1 \in N_{P}^{g}$ where 
$r / 1 \in h\left(R_{P}^{g}\right)$ and $m / 1 \in h\left(M_{P}^{g}\right)$. Thus $m / 1 \in N_{P}^{g}$ or $r / 1 \in\left(N_{P}^{g}: M_{P}^{g}\right)=\left(N:_{R} M\right)_{P}^{g}=P R_{P}^{g}$ since $N_{P}^{g}$ is a graded prime. Hence $r \in(N: M)=P$ or $m \in N$. Therefore, $N$ is a graded $P$-prime submodule of $M$.

\section{Graded weak multiplication modules}

Definition 3.1. Let $M$ be a graded $R$-module. $M$ is called a graded weak multiplication module if $G \operatorname{Spec}_{R} M=\varnothing$ or for every graded prime submodule $N$ of $M$, we have $N=I M$ where $I$ is a graded ideal of $R$.

One can easily show that if $M$ is a graded weak multiplication module, then $N=(N: M) M$ for every graded prime submodule $N$ of $M$.

Theorem 3.2. A graded $R$-module $M$ is a graded weak multiplication module if and only if the graded $R_{P}$-module $M_{P}$ is a graded weak multiplication module for every graded prime (or graded maximal) ideal $P$ of $R$.

Proof. Let $M$ be a graded weak multiplication $R$-module and $K$ a graded prime submodule of $M_{P}$ where $P$ is a graded prime ideal of $R$. So $K=N_{P}^{g}$ for some graded prime submodule $N$ of $M$. So $N=I M$ for some graded ideal $I$ of $R$. Hence $K=N_{P}^{g}=(I M)_{P}^{g}=I_{P}^{g} M_{P}^{g}$ because if $x / s \in$ $(I M)_{P}^{g}$, then $x / s=a m / t$ for some $a \in I, m \in M$ and $t \in S=R-P$, then $x / s=a / t . m / t \in I_{P}^{g} M_{P}^{g}$, so $(I M)_{P}^{g} \subseteq I_{P}^{g} M_{P}^{g}$, and clearly, $I_{P}^{g} M_{P}^{g} \subseteq(I M)_{P}^{g}$, so $(I M)_{P}^{g}=I_{P}^{g} M_{P}^{g}$. Thus $M_{P}^{g}$ is a graded weak multiplication $R_{P}^{g}$-module.

Conversely, let $N$ be a graded prime submodule of $M$. We show that $(N /(N: M) M)_{P}^{g}=0$ for every graded maximal ideal $P$ of $R$. If $(N: M) \subseteq P$, then by Theorem $2.4, N_{P}^{g}$ is a graded prime submodule of $M_{P}^{g}$. So $N_{P}^{g}=\left(N_{P}^{g}: M_{P}^{g}\right) M_{P}^{g}$, and by Lemma $2.3,\left(N_{P}^{g}: M_{P}^{g}\right)=(N: M)_{P}^{g}$. Hence $(N /(N: M) M)_{P}^{g}=N_{P}^{g} /\left(N:_{R} M\right)_{P}^{g} M_{P}^{g}=N_{P}^{g} /\left(N_{P}^{g}:_{R_{P}^{g}} M_{P}^{g}\right) M_{P}^{g}=0$. If $(N: M) \nsubseteq P$, then clearly $N_{P}^{g}=M_{P}^{g}$ and $(N: M)_{P}^{g}=R_{P}^{g}$. So $(N /(N: M) M)_{P}^{g}=N_{P}^{g} /(N: M)_{P}^{g} M_{P}^{g}=M_{P}^{g} / M_{P}^{g}=0$. Hence $(N /(N: M) M)_{P}^{g}=0$ for every graded maximal ideal $P$ of $R$. So $N /(N: M) M=0$, as needed.

Remark 3.3. Let $R$ be a graded integral domain. The quotient field $Q$ of $R$ is defined by $Q=$ $S^{-1} R$ where $S=h(R)-\{0\}$. Indeed $Q=\bigoplus_{g \in G} Q_{g}$ where $Q_{g}=\{r / s: r, s \in h(R), s \neq 0$ and $g=$ $\left.(\text { deg } s)^{-1}(\operatorname{deg} r)\right\}$ that is a graded field which is called the graded quotient field.

Definition 3.4. Let $R$ be a graded domain with the graded quotient field $Q$, the $\operatorname{Grank}(M)$ is defined to be the maximal number of homogeneous elements of $M$ linearly independent over $M$. In fact, $\operatorname{Grank}(M)$ is the dimension of the graded vector space $Q M$ over graded field $Q$, that is $\operatorname{Grank}(M)=\operatorname{Grank}_{Q}(Q M)$. 
Theorem 3.5. Let $M$ be a graded weak multiplication module over a graded domain, then

(i) If $M$ is a non-zero graded torsion free module, then $\operatorname{Grank}(M)=1$.

(ii) If $M$ is a graded torsion module, then $\operatorname{Grank}(M)=0$.

(iii) $M$ is either graded torsion or graded torsion free.

Proof. (i) First, Let $R$ is a graded field, so $0 \neq M$ is a graded vector space which is a graded weak multiplication module. If $\operatorname{Grank}(M)>1$, then let $0 \neq W \subset M$. According to Theorem 2.2, $W$ is a graded prime submodule of $M$, so $W=I M$ for some graded ideal $I$ of the graded field $R$. So $I=0$ or $I=R$, a contradiction. Hence $\operatorname{Grank}(M) \leq 1$, and since $M \neq 0$, then $\operatorname{Grank}(M)=1$. Now let $M$ be a non-zero graded torsion free $R$-module, then $Q M \neq 0$, which $Q$ is a graded quotient field of $R$. By Theorem 3.2, $Q M$ is a graded weak multiplication $Q$-module, and since we have proved in above $\operatorname{Grank}_{Q}(Q M)=1$, so $\operatorname{Grank}(M)=\operatorname{Grank}_{Q}(Q M)=1$.

(ii) Suppose that $M$ is a graded torsion module, then $Q M=0$ and therefore $\operatorname{Grank}(M)=$ $\operatorname{Grank}_{Q}(Q M)=0$.

(iii) Let $T(M) \neq M$. Then $T(M)$ is a graded prime submodule and $(T(M): M)=0$ by [1, Proposition 2.5]. Therefore since $M$ is graded weak multiplication, we have $T(M)=(T(M)$ : $M) M=0$, so the proof is complete.

A graded module $M$ over a $G$-graded ring $R$ is called locally graded cyclic if $M_{P}^{g}$ is graded cyclic $R_{p}^{g}$-module for all graded maximal ideal $P$ of $R$.

A graded module $M$ over a $G$-graded ring $R$ is called to be graded finitely generated if $M=\sum_{i=1}^{n} R x_{g_{i}}$ where $x_{g_{i}} \in h(M)$. A graded $R$-module $M$ is called graded cyclic if $M=R x_{g}$ where $x_{g} \in h(M)$.

Theorem 3.6. A graded finitely generated module is a graded multiplication module if and only if it is locally graded cyclic.

Proof. See [4, Proposition 2.4].

Theorem 3.7. Every graded finitely generated graded weak multiplication module is graded multiplication module.

Proof. Suppose that $M$ is a graded finitely generated graded weak multiplication $R$-module. We show that $M$ is locally graded cyclic, and by Theorem 3.5, $M$ is a graded multiplication module. By localization and Theorem 3.2, we can assume that $M$ is a graded finitely generated graded weak multiplication $R$-module where $R$ is a graded local ring. Let $m$ be the only graded maximal ideal of $R$. Obviously $M / m M$ is a graded finitely generated graded weak multiplication $R / m$-module. If $M=m M$, then by Nakayama Lemma for graded case (see [4]), we have $M=0$, so it is graded cyclic. 
If $m M \neq M$, then $\operatorname{Grank} k_{R / m}(M / m M)=1$, by Theorem 3.5 (i), so $M$ is graded cyclic.

The graded dimension of a $G$-graded $R$ is the supremum of the lengths of all chains of graded prime ideals in $R$ and denoted by $\operatorname{Gdim}(R)$.

Theorem 3.8. Let $R$ is a graded domain. Then the following are equivalent.

(i) $\operatorname{Gdim}(R)=0$.

(ii) For every graded weak multiplication $R$-module $M$, if $T(M)=0$, then $M$ is graded cyclic.

(iii) For every graded weak multiplication $R$-module $M$, if $T(M)=0$, then $M$ is graded multiplication.

Proof. (i) $\Rightarrow$ (ii) First let $R$ be a graded field. Let $M$ a graded torsion-free graded weak multiplication $R$-module. If $M=0$, then $M$ is graded cyclic. So let $M \neq 0$. Hence $M$ is a non-zero graded weak multiplication vector space over the graded field $R$. By Theorem 3.5, Grank $(M)=$ 1. That is $M \cong R$, so $M$ is graded cyclic.

Now we prove the general case. Let $0 \neq M$. By [1, Proposition 2.5], $T(M)=0$ is a graded prime submodule of $M$. Hence $(T(M): M)$ is a graded prime ideal of $R$ and since $\operatorname{Gdim}(R)=$ $0, R /(T(M): M)$ is a graded field. Since $T(M)=0$, so $M \cong M / 0=M / T(M)$ is a graded torsionfree graded weak multiplication $R /(T(M): M)$-module. So $M$ is a graded torsion-free graded weak multiplication module over a graded field $R /(T(M): M)$. Therefore as we have proved in above $M$ is a graded cyclic $R /(T(M): M)$-module and by the fact $(r+(T(M): M)) m=r m$ for all $r \in R$ and $m \in M$, then $M$ is a graded cyclic $R$-module.

(ii) $\Rightarrow$ (iii) By [4, Proposition 2.4]

(iii) $\Rightarrow$ (i) Let $P$ be a graded prime ideal of $R$. It is enough to prove that $R / P$ is a graded field. If $Q$ is the graded quotient field of $R$, then 0 is the only graded prime ideal of $R / P$-module $Q$. Hence $Q$ is a graded torsion-free graded weak multiplication $R / P$-module. Therefore by assumption $Q$ is a graded multiplication module. Since $R / P$ is a graded submodule of $Q$, so $R / P=I Q$ for some graded ideal $I$ of $R / P$. Clearly, $I Q=Q$, so $Q=R / P$, hence $P$ is a graded maximal ideal of $R$. Therefore, $\operatorname{Gdim}(R)=0$.

Corollary 3.9. Let $R$ is a graded integral domain. Then the following are equivalent.

(i) $R$ is a graded field.

(ii) Every graded weak multiplication $R$-module is graded cyclic.

(iii) Every graded weak multiplication $R$-module is graded multiplication.

Proof. If $R$ is graded field, and since every graded weak multiplication $R$-module is a graded vector space, it is a graded torsion-free graded weak multiplication $R$-module, so the proof follow by Theorem 3.8. 
Corollary 3.10. If $m$ is a graded maximal ideal of the graded ring $R$ which is a minimal graded prime ideal and $m \neq m^{2}$, then the following are equivalent.

(i) $m$ is a graded weak multiplication $R$-module.

(ii) There is no graded ideal between $m^{2}$ and $m$.

(iii) $\operatorname{GSpec}_{R} m=\left\{m^{2}\right\}$.

Proof. By localization and Theorem 3.2 we can assume that $R$ is a graded local ring with the only graded prime ideal $m$.

(i) $\Rightarrow$ (ii) Let $m$ be a graded weak multiplication $R$-module. If $m^{2} \subseteq I \subseteq m$ where $I$ is graded ideal of $R$, we show that $I$ is a graded prime submodule of $m$. Let $r a \in I$ where $r \in h(R)$ and $a \in h(m)$. Suppose that $a \notin I$, then $r$ is not unit, hence $r \in m$. Therefore $r m \subseteq m^{2} \subset I$, that is $I$ is a graded prime submodule of $m$.

Since $m$ is graded weak multiplication module, and $I$ a graded prime submodule, then $I=m m_{1}$ for some graded ideal $m_{1}$ of $R$. If $m_{1}=R$, then $I=m R=m$, which is a contradiction. So $m_{1} \subseteq m$, that is $m^{2} \subseteq I=m m_{1} \subseteq m^{2}$, thus there is no graded ideal between $m^{2}$ and $m$.

(ii) $\Rightarrow$ (iii) Suppose that there is no graded ideal between $m^{2}$ and $m$. If $I$ is a graded prime submodule of the graded $R$-module $m$, then $(I: m)$ is a graded prime ideal by [3, Proposition 2.5]. Since $m$ is the only graded prime ideal of $R$, then $(I: m)=m$. Therefore, $m^{2} \subseteq I \subseteq m$, and by assumption $I=m^{2}$, hence $G \operatorname{Spec}_{R} m=\left\{m^{2}\right\}$.

\section{Acknowledgement}

I would like to thank the referee(s) for his comments and suggestions on the manuscript.

\section{References}

[1] S. Ebrahimi Atani, On graded prime submodules, Chiang Mai J . Sci. , 2006,33(1), 3-7.

[2] S. Ebrahimi Atani and F. Farzalipour, Notes On the graded prime submodules, Int. Math. Forum 1(2006), 18711880.

[3] S. Ebrahimi Atani and F. Farzalipour, On graded secondary modules, Tuikish. J . Math. 31(2007), 371-378.

[4] P. Ghiasvand and F. Farzalipour, Some Properties of Graded Multiplication Modules, Far East J . Math. Sci. 34(2009), 341-352.

[5] C. Nastasescu and F. van Oystaeyen, Graded Rings Theory, Mathhematical Library 28 , North Holand, Amsterdam, 1982.

[6] M. Refaei and K. Alzobi, On graded primary ideals, Turkish. J . Math. 28(2004), 217-229.

Department of Mathematics, Payame Noor University, Tehran 19395-3697, Iran.

E-mail: f.farzalipour@pnu.ac.ir

Department of Mathematics, Payame Noor University, Tehran 19395-3697, Iran.

E-mail: p.ghiasvand@pnu.ac.ir 